Research article

\title{
Simultaneous determination of shikimic acid, salicylic acid and jasmonic acid in wild and transgenic Nicotiana langsdorffii plants exposed to abiotic stresses
}

\author{
Elisa Scalabrin ${ }^{\text {a, }}$, Marta Radaelli ${ }^{\text {b }}$, Gabriele Capodaglio ${ }^{\text {a, b }}$ \\ a Department of Environmental Sciences, Informatics and Statistics, University of Venice, Ca' Foscari, Via Torino 155, 30172 Venice, Italy \\ ${ }^{\mathrm{b}}$ Institute for the Dynamics of Environmental Processes-CNR, University of Venice, Via Torino 155, 30172 Venice, Italy
}

\section{A R T I C L E I N F O}

\section{Article history:}

Received 18 December 2015

Received in revised form

18 February 2016

Accepted 26 February 2016

Available online 2 March 2016

\section{Keywords:}

Abiotic stress

Jasmonic acid

Nicotiana langsdorffii

Rat glucocorticoid receptor gene

rol C

Salicylic acid

Shikimic acid

\begin{abstract}
A B S T R A C T
The presence and relative concentration of phytohormones may be regarded as a good indicator of an organism's physiological state. The integration of the rolC gene from Agrobacterium rhizogenes and of the rat glucocorticoid receptor ( $g r$ ) in Nicotiana langsdorffii Weinmann plants has shown to determine various physiological and metabolic effects. The analysis of wild and transgenic N. langsdorffii plants, exposed to different abiotic stresses (high temperature, water deficit, and high chromium concentrations) was conducted, in order to investigate the metabolic effects of the inserted genes in response to the applied stresses. The development of a new analytical procedure was necessary, in order to assure the simultaneous determination of analytes and to obtain an adequately low limit of quantification. For the first time, a sensitive HPLC-HRMS quantitative method for the simultaneous determination of salicylic acid, jasmonic acid and shikimic acid was developed and validated. The method was applied to 80 plant samples, permitting the evaluation of plant stress responses and highlighting some metabolic mechanisms. Salicylic, jasmonic and shikimic acids proved to be suitable for the comprehension of plant stress responses. Chemical and heat stresses showed to induce the highest changes in plant hormonal status, differently affecting plant response. The potential of each genetic modification toward the applied stresses was marked and particularly the resistance of the gr modified plants was evidenced. This work provides new information in the study of $N$. langsdorffii and transgenic organisms, which could be useful for the further application of these transgenes.
\end{abstract}

(C) 2016 Elsevier Masson SAS. All rights reserved.

\section{Introduction}

When in adverse or limiting conditions, plants activate a complex system of responses in order to alleviate cellular damage and to survive (Fuoco et al., 2013). Water deficiency, high temperatures and pollution represent the main stress factors for plants in relation to the expected climate changes. Heat stress conditions affect the cell membranes and the enzyme functionality while modifying the

Abbreviations: ABA, Abscisic acid; ACN, acetonitrile; Cr, chromium; gr, glucocorticoid receptor; $\mathrm{HS}$, heat stress; IS, internal standard; FR, instrumental response factor; JA, jasmonic acid; LOD, limit of detection; LOQ limit of quantification; ME, matrix effect; $\mathrm{MeOH}$, methanol; PTFE, polytetrafluoroethylene; PEG 6000, polyethylene glycol 6000; RSA, radical scavenging activity; ROS, reactive oxygen species; SA, salicylic acid; $S^{13} C_{6}$, salicylic acid phenyl ${ }^{13} C_{6}$; SHA, shikimic acid; WS, water stress; WT, wild type.

* Corresponding author.

E-mail address: elisa.scalabrin@unive.it (E. Scalabrin). transpiration rate (Lipiec et al., 2013); water deficiency determines the inhibition of photosynthesis, the enhancement of respiration and the lack of mineral nutrients (Yordanov et al., 2000). Heavy metals, due to their widespread distribution and their persistency, represent one of the main issues for agriculture and land use. Heavy metals such as cadmium and chromium $(\mathrm{Cr})$ induce enzyme inhibition, cellular oxidation and the alteration of metabolism (Obata and Fernie, 2012). $\mathrm{Cr}(\mathrm{VI})$ is the most toxic oxidation state of chromium, whose uptake was shown to influence the plant's growth, the production of many essential metabolites and enzymatic activity (Singh et al., 2015).

The use of genetic engineering to produce transformed stressresistant organisms is increasingly gaining interest. Among the genetic modifications studied, the integration of the gene codifying for the rat glucocorticoid receptor ( $g r$ ), which regulates genes controlling the development, metabolism and immune response, appears to be promising, inducing higher resistance against 
nematode infections and chemical stress in Nicotiana plants (Del Bubba et al., 2013). The rolC gene is a plant oncogene carried on plasmids of the plant pathogen Agrobacterium rhizogenes; after infection, the gene can be transferred to the plant genome causing hairy root disease and tumor formation. Multiple biochemical and physiological alterations have been observed in rolC transformed plants, including stimulation of alkaloid, anthraquinone and cytokinin production (Bulgakov et al., 2008; Kiselev et al., 2006). Enhancement of plant response to abiotic and biotic stresses has even been related with rolC gene insertion (Del Bubba et al., 2013; Intrieri and Buiatti, 2001). The Nicotiana genus (family of Solanaceae) includes small, well-characterized plants, traditionally used as biological models for genetic and physiological studies; the genetic rolC and gr modifications of Nicotiana langsdorffii plants were previously investigated, yielding interesting results for the production of plants resistant to different stresses (Del Bubba et al., 2013; Fuoco et al., 2013; Ranaldo et al., 2015). The biological state of plants can be monitored through different parameters such as their morphology, anatomy, physiology and biochemistry. Since the response to environmental stresses is controlled by the hormonal network, the presence and relative concentration of hormones may be regarded as a good indicator of an organism's physiological condition. Among the complex hormonal signaling system of plants, the following molecules have been recognized as central components of the adaptation response.

Salicylic acid (SA) and jasmonic acid (JA) are hormones involved in plant growth and development; recent studies demonstrated that they are implicated as signaling compounds in response to both biotic and abiotic stresses (Clarke et al., 2009; Maksymiec, 2007; Maksymiec et al., 2005; Metwally et al., 2003; Pál et al., 2005); many studies showed that the relative concentrations of JA and SA are affected during drought, chemical and temperature stresses (Clarke et al., 2009; De Ollas et al., 2013; He et al., 2014; Maksymiec et al., 2005; Pál et al., 2005; Wang et al., 2010). Shikimic acid (SHA) is an important intermediate in plant metabolism and a key molecule in the biosynthesis of numerous secondary metabolites. The SHA pathway represents the central point for the production of many compounds involved in the principal functions of plant life, including defense, such as flavonoids, lignins, indole derivatives and many aromatic alkaloids. The SHA pathway leads also to the production of SA, through the first step of the phenylpropanoid pathway or directly from isochorismate. The targeted determination of these three compounds could be very useful in the investigation of the effects of rolC and $g r$ insertion in $N$. langsdorffii and in the better comprehension of plant response towards abiotic stresses. The aim of this paper is to investigate the effects of $\mathrm{Cr}(\mathrm{VI})$ exposure, water deficiency and high temperature on wild and transgenic $N$. langsdorffii plants, through the analysis of selected metabolites, in order to highlight the influence of the inserted transgenes (rolC and gr genes) on plant stress responses. The morphological and physiological effects of rolC and gr insertion in $N$. langsdorffii plants, exposed to heat, water and chemical stresses, have been the subject of other studies (Bogani et al., 2015; Ancillotti et al., 2015).

Taking advantage from the use of HPLC-HRMS technology, the quantitative determination of SA, SHA and JA was performed. The development of a new analytical procedure was necessary, in order to assure the simultaneous determination of analytes, due to the limited available plant material, and to obtain a limit of quantification adequately low to fulfill the analyte concentrations. The use of a high-resolution detector permitted the accurate measurement of metabolite masses and the discrimination between the analytes and potential interfering compounds; therefore the sample treatment procedure was fast, not requiring the purification step, which is generally essential for biological matrices analyses.
To our knowledge, no method for the simultaneous determination of these three compounds has been reported yet. The comparative evaluation of phytohormonal changes, induced by different abiotic stress factors, in wild type and in $\mathrm{gr}$ and rolC plants, allowed us to study the different metabolic mechanisms involved in stress response, in order to identify the organisms more promisingly resistant to the applied stresses.

\section{Material and methods}

\subsection{Chemicals}

SHA, JA, SA, salicylic acid phenyl ${ }^{13} \mathrm{C}_{6}\left(\mathrm{SA}^{13} \mathrm{C}_{6}\right)$ and acetic acid HPLC grade were purchased from Sigma Aldrich ${ }^{\circledR}$ (Buchs, Switzerland). HPLC/MS-grade methanol (MeOH) and acetonitrile (ACN) were obtained from Romil LDT (Cambridge, U.K.). Hydrochloric acid $(\mathrm{HCl}) 37 \%$ ACS was purchased from Carlo Erba Reagents (Milano, Italy). Ultrapure water (18.2 $\mathrm{M} \Omega \mathrm{cm}, 0.01 \mathrm{TOC}$ ) was produced using a Purelab Ultra System (Elga, High Wycombe, U.K.).

\subsection{Stock and working solutions}

Stock standard solutions $(10 \mu \mathrm{g} / \mu \mathrm{L})$ of SHA, JA, SA and $\mathrm{SA}^{13} \mathrm{C}_{6}$ were prepared in $A C N$. Working standard solutions were prepared by diluting the stock solutions to obtain concentrations of $0.97 \mathrm{ng} /$ $\mu \mathrm{L}$ for $\mathrm{SA}^{13} \mathrm{C}_{6}$ and $10 \mathrm{ng} / \mu \mathrm{L}$ for SA, SHA and JA.

\subsection{Plant material}

Plants of $N$. langsdorffii Weinmann were cultivated in vitro by Dr. Patrizia Bogani in the Laboratory of Plant Genetics, Department of Evolutionary Biology of the University of Florence. Wild type plants (WT) were genetically modified by inserting two kinds of genes: the gene codifying for the rat glucocorticoid receptor ( $g r$ plants) and the rolC gene from $A$. rhizogenes (rolC plants). The procedure for obtaining these genetic modifications is well described in previous studies (Del Bubba et al., 2013; Fuoco et al., 2013). Genetic identical plants were obtained by withdrawing portions of stems containing the internodes. Each plant was screened for the presence and the expression of $g r$ and rolC genes as previously described (Fuoco et al., 2013). Plants were grown in vitro until reaching 30 days, as earlier explained (Del Bubba et al., 2013; Fuoco et al., 2013; Giannarelli et al., 2010). Prior to analysis, plants were fast cleaned with distilled water to remove the LS medium residues and frozen in liquid nitrogen; the whole plants were then freeze-dried in an Edward machine and, after complete water evaporation, maintained at environmental temperature.

\subsection{Stress inductions}

The plants analyzed in this study were subjected to heat stress (HS) by means of the heat shock method, through exposure at $50^{\circ} \mathrm{C}$ for $2 \mathrm{~h}$ inside a thermostatic chamber; the water stress (WS) was induced by subculturing plants for 15 days on $50 \mathrm{~mL}$ of LS medium conditioned with $50 \mathrm{~mL}$ of a $20 \%$ polyethylene glycol 6000 (PEG $6000)$ solution, in accordance with literature (Khalid et al., 2010). These conditions were selected after test studies, as previously explained (Ancillotti et al., 2015; Scalabrin et al., 2015). The heavy metal stress by chromium $(\mathrm{Cr})$ was induced by growing plants for 15 days using an LS medium containing $\mathrm{K}_{2} \mathrm{Cr}_{2} \mathrm{O}_{7}(50 \mathrm{mg} / \mathrm{kg}$ of hexavalent chromium), as described by Del Bubba et al., 2013 and on the basis of preliminary stress responses experiments (Fuoco et al., 2013). 


\subsection{Sample treatment protocol}

Globally, 80 samples of $N$. langsdorffii $(g r=10, \mathrm{wt}=10$, rolC $=10$, $g r-\mathrm{WS}=6, \mathrm{wt}-\mathrm{WS}=5$, rolc-WS $=4, \mathrm{wt}-\mathrm{Cr}=10, \mathrm{gr}-\mathrm{Cr}=11$, rolc$\mathrm{Cr}=7$, WT-HS $=3$, rolc-HS $=2, g r-\mathrm{HS}=2$ ) were analyzed; the number of samples for each genotype and stress depended on the availability of plant material (supplied by the University of Florence) and on the weight of plants. With respect to HS plants, due to the limited number ( 3 organisms for each genotype) and weight of these plants, all the available plant material was mixed, obtaining an homogenized pool which was analyzed in three (wt-HS) or two (rolc-HS, gr-HS) independent technical replicates. For phytohormone analysis, samples were ground and homogenized by using a ball mill equipped with two polytetrafluoroethylene (PTFE) vessels and grinder balls; samples were ground for 5 min with a vibration frequency of $20 \mathrm{~Hz}$ to achieve a final fineness of $\approx 5 \mu \mathrm{m}$. Briefly, $0.1 \mathrm{~g}$ of homogenized plant material was weighted and the internal standard (IS) $\mathrm{SA}^{13} \mathrm{C}_{6}$ was added. The samples were extracted three times with $1.5 \mathrm{~mL}$ of fresh $\mathrm{MeOH}$ acidified with $\mathrm{HCl}(99.9 / 0.1, \mathrm{v} / \mathrm{v})$ by centrifugation for $20 \mathrm{~min}$ at 20,000 rcf (relative centrifugal force) at $25{ }^{\circ} \mathrm{C}$; the supernatants were collected, unified and evaporated in a thermostated bath at $30{ }^{\circ} \mathrm{C}$, under a gentle stream of nitrogen. Once a $0.5 \mathrm{~mL}$ was reached, $1 \mathrm{~mL}$ of water was added and the extracts were filtrated with a syringe PTFE filter $(\varnothing 25 \mathrm{~mm}$, $0.45 \mu \mathrm{m})$. Two fractions were prepared for LC-MS analysis by diluting the extracts 1 to 20 times for SHA and SA determination and 1 to 5 times for JA. Along with each set of samples, one blank sample (for a total amount of eight blanks) was analyzed; the blanks were subjected to all processing steps, in order to verify the eventual contribution to analyte concentrations deriving from sample handling; moreover, blanks were used to calculate the limits of detection and quantification (LOD and LOQ) of the analytical procedure. SA and JA were quantified by means of the IS method while SHA was determined by means of an external calibration curve (see Appendix A, paragraph S2, Table S1). For this reason, for each sample batch, a calibration curve of SHA was also analyzed, in order to effectuate a correct quantification and to minimize instrumental signal variations. All samples were analyzed on the same day of their extraction, to prevent compound degradation.

\subsection{Instrumental method}

The instrumental analyses were carried out on a UHPLC Dionex Ultimate 3000 LC system coupled to an HESI-LTQ Orbitrap (Thermo Fisher Scientific, Waltham, USA). The analysis was conducted with the ESI ion source operating in negative polarity, in full scan mode at the resolving power of 30,000 full width at half maximum. The final instrumental selected parameters are reported in Appendix A, paragraph S1, Table S2. The quantification of SA and JA was performed by means of an instrumental response factor (FR) to reduce possible instrumental signal variations. The main analytical parameters for each compound are reported in Table 1. The chromatographic separation was performed on a $C_{18}$ phase $4 \mu \mathrm{m}$ Synergy Hydro-RP $80 \AA$, $50 \times 2 \mathrm{~mm}$ (Phenomenex ${ }^{\circledR}$, Torrance, California, USA) eluted with acetic acid $0.1 \%$ (Eluent A) and $\mathrm{MeOH}$
(Eluent B) at a flow rate of $200 \mu \mathrm{L} / \mathrm{min}$. The column oven temperature was set at $30{ }^{\circ} \mathrm{C}$. The selected chromatographic run was composed of an initial 2 min isocratic step (100\% eluent A) for the elution of SHA, followed by a $5 \mathrm{~min}$ gradient phase $(20 \% / \mathrm{min})$ until reaching $100 \%$ of eluent $B$. The elution of $S A, S A^{13} C_{6}$ and JA was obtained with a following isocratic phase (100\% eluent B) of $3 \mathrm{~min}$ (Fig. 1); the final step, for column conditioning, takes $7 \mathrm{~min}$ at $100 \%$ eluent $A$.

\subsection{Method validation (see Appendix A paragraph S2, Table S1)}

The method was validated by verifying accuracy, precision, analyte recovery and instrumental linearity. The matrix effect (ME) was also estimated, in order to assess the most appropriate quantification method.

\subsection{Data processing and statistics}

Chromatograms were integrated with LCquant 2.6.1 software (Thermo Fisher Scientific, Waltham, USA). The extracted ion chromatograms were generated by considering a small mass range $( \pm 5 \mathrm{ppm})$, centered on the exact $\mathrm{m} / \mathrm{z}$ of each analyte. Data elaborations were performed by Excel add-in Multibase 2015 package (Numerical Dynamics, Japan). Statistical differences between controls and treated samples were analyzed using Student's t-test by means of Statistica 8.0 (StatSoft, Inc., 2007). Differences were considered significant at a probability level of $p<0.05$.

\section{Results and discussion}

\subsection{Effect of rolC and gr insertion}

The gr-modified plants were obtained by inserting a rat gene encoding the glucocorticoid receptor, containing the constitutive CaMV promoter; it is possible that plant phyto-steroids, and particularly brassinosteroids, which showed to induce tolerance towards oxidative, drought and heavy metal stresses (Bartwal et al., 2013), have sufficient affinity with the rat glucocorticoid receptor protein and, therefore, can be activated by this signaling chain in $g r$ transgenic plants. The $g r$ insertion in $N$. langsdorffii has shown to determine the reduction of plant size, the decrease of electrolyte leakage and the increase of water content (Bogani et al., 2015). The integration of the rolC gene in plants reduced plant size, electrolyte leakage and root and shoot dry biomass (Ancillotti et al., 2015), while increased phytoalexin production and the expression of pathogenesis-related proteins (Bulgakov et al., 2008); rolC insertion, moreover, has been related to an increased activity of cytokinins and to the induction of many secondary metabolite pathways (Kiselev et al., 2006).

In this study, the insertion of rolC gene affected the content of JA, which was reduced in this genotype in comparison to $\mathrm{gr}$ and $\mathrm{wt}$ plants. This result could be related to the ability of cytokinins to suppress the JA levels, in agreement with the observations of Sano et al., 1996. As expected, JA showed lower concentrations $\left(1 \times 10^{1}-1.8 \times 10^{2} \mathrm{ng} / \mathrm{g}\right.$ d.w. $)$ than SA and SHA $\left(3 \times 10^{3}\right.$ to $1.7 \times 10^{4} \mathrm{ng} / \mathrm{g}$ d.w. and $2.5 \times 10^{3}$ to $5.5 \times 10^{3} \mathrm{ng} / \mathrm{g}$ d.w.

Table 1

Accurate masses, ionization modus, internal standard used, retention time and maximum mass deviation of the analytes.

\begin{tabular}{|c|c|c|c|c|c|}
\hline Compounds & Ionization modus & Retention time (min) & Accurate mass $(m / z)$ & Internal standard & Mass deviations (ppm) \\
\hline Shikimic acid & negative & 1.05 & 173.0455 & Not used & -2.88 \\
\hline Salicylic acid & negative & 7.16 & 137.0244 & $\mathrm{SA}^{13} \mathrm{C}_{6}$ & -1.45 \\
\hline Jasmonic acid & negative & 7.83 & 209.1183 & $\mathrm{SA}^{13} \mathrm{C}_{6}$ & -2.86 \\
\hline
\end{tabular}




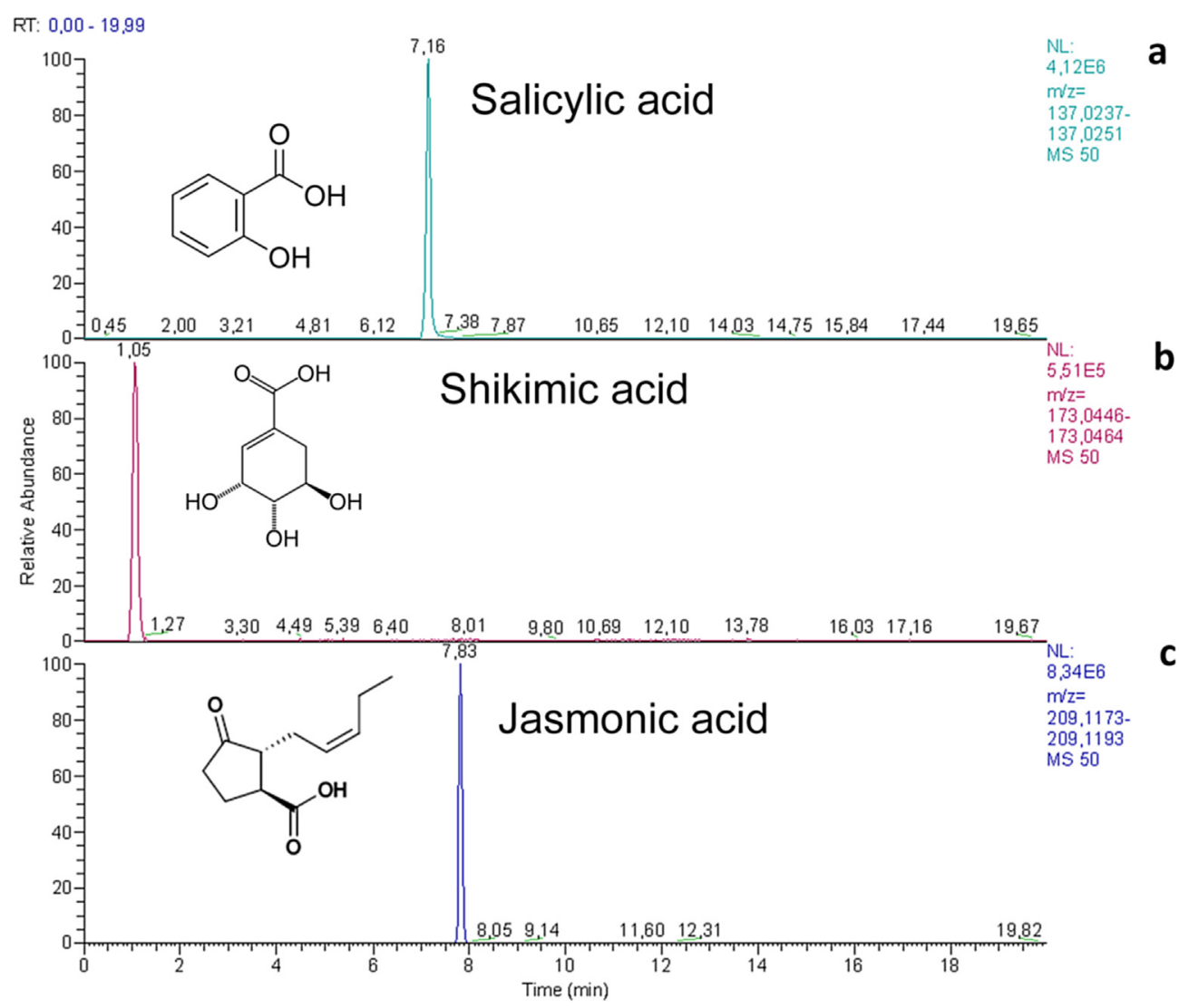

Fig. 1. Chromatographic separation of SA (a), SHA (b) and JA (c) in matrix enriched with standard solutions of analytes, at the concentration level of 50 pg/ $\mu \mathrm{L}$.

respectively). JA had the highest internal set variability, with relative standard deviations (RSD \%) ranging from 34 in rolC plants to 66 in $g r$ plants (Appendix A, Table S3). SA (RSD\% = 32-34) and SHA (RSD\% $=16-23)$ showed no statistically significant differences among the genotypes. The results are in agreement with previous studies (Schmelz et al., 2003; Fuoco et al., 2013). Interestingly, the concentration of SHA was similar in wt, gr and rolC plants, despite other studies suggested an increase of phenolic compound concentrations in transgenic plants (Del Bubba et al., 2013; Ancillotti et al., 2015); this finding indicates a different regulation of phenolics' biosynthesis in $g r$ and rolC genotypes, probably through a modification of the phytohormonal balance in these organisms (Giannarelli et al., 2010).

\subsection{Heat stress (HS)}

The highest changes in analyte concentrations between controls and stressed samples were observed in plants exposed to HS. JA levels were increased 2.1 and 6.8 fold in wt-HS and rolC-HS plants respectively, while $g r-\mathrm{HS}$ plants showed unchanged concentrations of this compound.

The induction of JA has already been related with HS exposure, possibly caused by the disruption of cell membranes. Rizhsky et al., 2002, observed high lipoxygenase expression in Nicotiana tabacum during heat and drought stress, indicating the enhancement of JA biosynthesis. Endogenous JA biosynthesis and signaling, moreover, showed to be essential for thermotolerance in Arabidopsis thaliana (Clarke et al., 2009).

Interestingly, JA content in gr plants was not affected by HS exposure, suggesting a possible influence of this genetic modification on plant response toward heat stress. The unchanged concentrations of JA between $g r$ and $g r$-HS plants could indicate a lighter damage of the photosynthetic system and of the membrane structures in this genotype, as confirmed by the limited changes in the lipidic profile of $\mathrm{gr}$ plants in comparison to rolC and wt genotypes (Scalabrin et al., 2015). This resistance could be due to the higher level of abscisic acid (ABA) in the gr genotype (Giannarelli et al., 2010), which might determine an earlier and more effective activation of plant defenses against this kind of stress (Kazan, 2015) in comparison to wt and rolC.

The phytohormone SA is known to take part in the defense against temperature stress, which primarily compromises the photosynthesis process; SA particularly promotes the activity of the Rubisco enzyme and increases the efficiency of the photosynthetic system during heat stress exposure (Wang et al., 2010). However, several studies reported an initial substantial increase of endogenous SA, in the first 30 min of the HS application, followed by a drastic decrease, indicating that the accumulation of SA represents an early signal in heat response (Pan et al., 2006). Nevertheless, the induction of SA has been explained as an indirect effect of heat stress exposure, as a consequence of ABA increase (Kurepin et al., 2013); moreover Clarke et al., 2009 highlighted the synergic/ antagonistic interaction of SA and JA during heat stress.

In our study, $2 \mathrm{~h}$ exposure to $50{ }^{\circ} \mathrm{C}$ determined unchanged SA levels in rolC-HS and $g r$-HS plants, consistently with literature, while a significant decrease was observed in wt-HS plants (Fig. 2 a,b,c). SA decreased levels in wt plants could indicate a higher thermosensitivity of this genotype, in comparison to $\mathrm{gr}$ and $\mathrm{rolC}$, whose biochemical status showed to be less affected by heat stress (Scalabrin et al., 2015). However, the mechanisms of regulation of SA levels and the interactions with JA, ABA and other phytohormones are not fully understood yet. 
SA

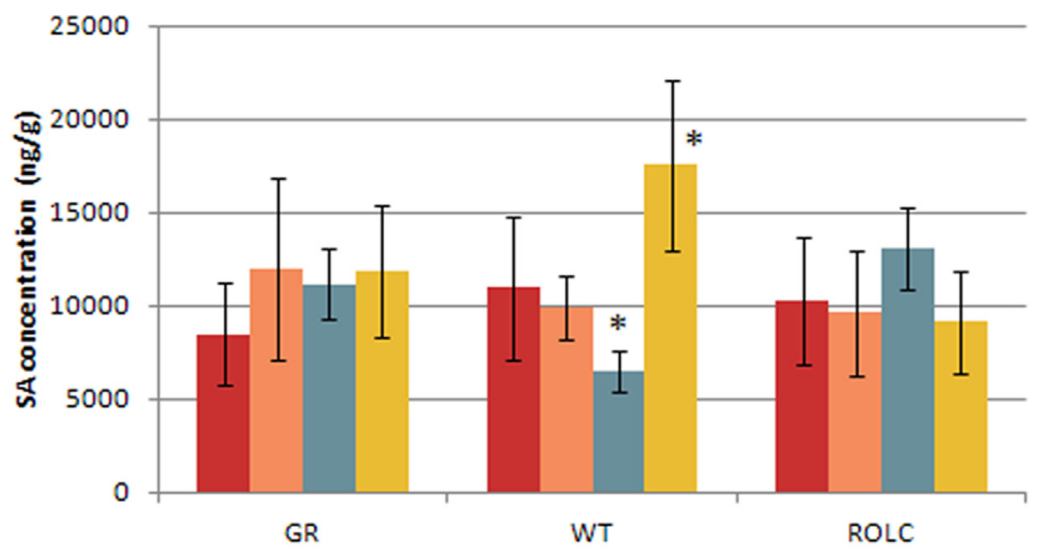

SHA

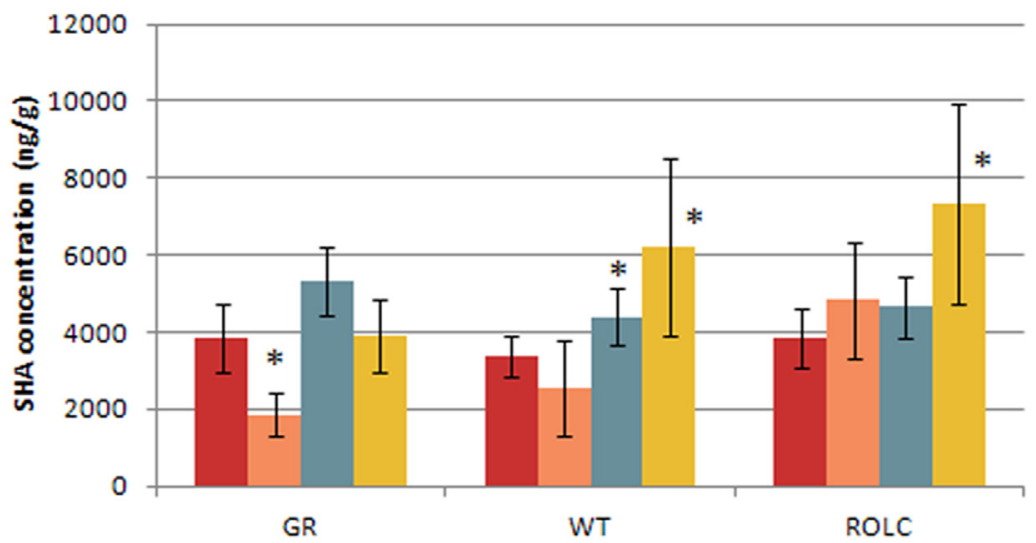

JA

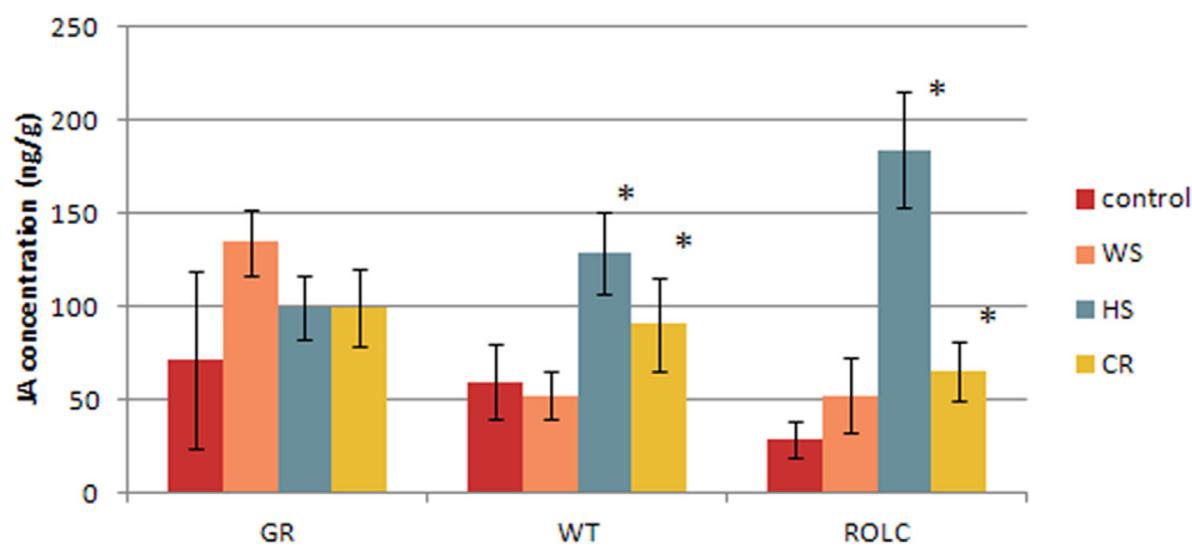

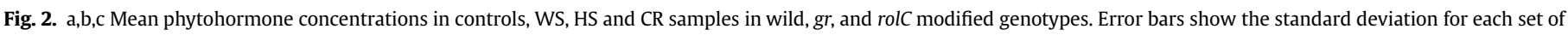

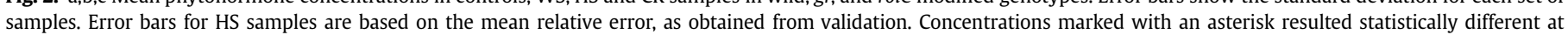
$\mathrm{p}<0.05$ according to the t-test.

HS is known to cause oxidative stress (Wang et al., 2010), generating reactive oxygen species (ROS) which compromise membrane fluidity, protein stability and enzymatic functions (Lipiec et al., 2013). To alleviate cellular damages and scavenge ROS compounds, plants produce antioxidant metabolites, such as flavonoids, anthocyanins, phenolics, whose biosynthesis starts from a

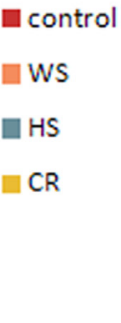

b

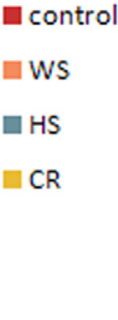

C

the SHA pathway (Lipiec et al., 2013; Obata and Fernie, 2012). Interestingly, in our study, SHA levels showed to increase only in wt-HS plants, indicating the activation of the shikimate metabolic route. This result is in agreement with the study of Ancillotti et al., 2015, who highlighted increased levels of total polyphenols and radical scavenging activity (RSA) in the shoots of wt-HS, while gr 
and rolC plants showed reduced antioxidants levels. It is noteworthy that in wt-HS plants the levels of the precursor SHA were increased while SA, on the contrary, was reduced. This finding could suggest that the raised SHA produced in wt-HS plants is not employed for the biosynthesis of SA whereas, probably, it is mainly re-routed in the phenylpropanoid pathway for the synthesis of antioxidant compounds, in order to face ROS molecules. RolC-HS plants showed unchanged SHA levels, confirming the previous result of Bulgakov et al., 2008, who reported the prevention of oxidative burst in Rubia cordifolia rolC transformed cells and their increased HS tolerance. With respect to gr plants, the unchanged SHA levels could indicate that the induction of this metabolic pathway during HS is not required in this genotype; the higher basal levels of hydroxycinnamic acids, which are the main precursors of phenolics, suggest an enhancement of defenses in this genotype that, in cases of abiotic stress exposure, proved to be already active, not requiring an higher induction of the SHA pathway (Del Bubba et al., 2013).

\section{3. $\mathrm{Cr}(\mathrm{VI})$ stress}

Oxidative stress is one of the most evident consequences of heavy metal exposure through the enhanced production of ROS (Maksymiec, 2007). Particularly, exposure to $\mathrm{Cr}(\mathrm{VI})$ largely increases ROS levels, activating a signaling response at gene expression level which could increase active oxidant scavenging (Singh et al., 2015). In case of severe chemical stress, lipid peroxidation and the generation of oxylipins, such as JA, can take place (Maksymiec, 2007). In our samples, we actually observed an enhancement of JA and SHA levels in wt-Cr and rolC-Cr, with a statistically significant increase twice as high as in the controls (Fig. 2 b,c), while gr-Cr showed no significant changes. The induction of the octadecenoic pathway, probably caused by the degradation of membranes (Maksymiec et al., 2005), was already observed after exposure to heavy metals, leading to the production of JA and other oxylipins (Maksymiec, 2007; Maksymiec et al., 2005). This pathway is part of the oxidative/redox system of plants and, therefore, probably interacts with metal induced signaling and even in potential defense reactions (Viehweger, 2014). JA could be involved in the upregulation of the genes related to the metabolism of glutathione, which is a key element of cellular redox homeostasis and has a defense role against oxidative stress (Xiang and Oliver, 1998). As already outlined for plants exposed to HS, SHA represents an indicator of the induction of antioxidant biosynthesis. SHA proved to be indeed enhanced in wt$\mathrm{Cr}$ and rolC-Cr samples, while it remained unchanged in $\mathrm{gr}-\mathrm{Cr}$, indicating a lower activation of the defense mechanisms in this genotype. Our results are in agreement with the recent study of Scalabrin et al., 2015 who reported increased levels of peroxylipids, lysolipids and antioxidant compounds in wt and rolC plants exposed to $\mathrm{Cr}(\mathrm{VI})$ stress while the levels of these metabolites were unchanged in the gr genotype. The SHA concentrations observed in this study are also consistent with the results of Fuoco et al., 2013 for wt and gr genotypes. The increase of SHA concentrations in rolC-Cr plants indicates that, differently from what is observed in rolC-HS, the ROS suppression effect is not effective against $\mathrm{Cr}(\mathrm{VI})$ stress. This fact is probably due to the complex action of heavy metals on the oxidative/redox system of plants, which includes the direct generation of ROS via Fenton-like reactions and the Haber-Weiss cycle, in addition to indirect mechanisms, and the consumption of glutathione as direct chelator for metal detoxification (Viehweger, 2014). This is also consistent with the enhanced concentration of JA in rolC-Cr samples. Interestingly, while in $\mathrm{wt}-\mathrm{Cr}$ plants the increased SHA levels lead also to enhanced concentrations of its derivative, SA, in rolC-Cr plants only SHA was augmented. In wt-Cr plants, a significant SA increase of $57 \%$ was observed, in comparison to controls, while in $\mathrm{gr}-\mathrm{Cr}$ and rolC-Cr SA levels were unchanged. The role of SA in heavy metal stress defense is currently not well understood; this compound showed to increase in Barley seedlings and maize plants subjected to high cadmium levels (Metwally et al., 2003; Pál et al., 2005) and in Oryza sativa spp. Japonica exposed to oxidative stress (Yang et al., 2004) while, in other studies, decreased or unchanged concentrations were observed (Singh et al., 2015). SA seems to increase oxidative stress by reducing the activity of phytochelatin synthase (Pál et al., 2002), increasing $\mathrm{H}_{2} \mathrm{O}_{2}$ levels (Zawoznik et al., 2007) and inhibiting the JA pathway (Maksymiec, 2007). The increase in SA concentrations could probably be considered as an early response to heavy metal stress, as already described for HS stress, which is needed in order to activate the plant response mechanisms (Zawoznik et al., 2007).

\subsection{Water stress (WS)}

In plants exposed to WS, a 50\% decrease of SHA concentrations was observed in $\mathrm{gr}-\mathrm{Cr}$ samples, in comparison to controls. The lower activation of SHA pathway in gr-WS plants than in rolC-WS and wt-WS genotypes was already reported (Scalabrin et al., 2015) and it's in accordance with the decreased RSA recently observed by Ancillotti et al., 2015. The plant's response to water deficit is complex and highly variable depending on the species, developmental stage, desiccation degree (Obata and Fernie, 2012), and interaction with other environmental factors, such as relative humidity and temperature. Different biochemical responses to water deficit conditions have even been observed in various varieties of the same species (Sánchez-Rodríguez et al., 2011). The shikimic acid pathway is generally induced by water stress exposition and different regulation mechanisms have been proposed, such as the maintenance of the osmotic pressure level (Warren et al., 2012), the need for increased antioxidant activity (Sánchez-Rodríguez et al., 2011) and the decreased lignification degree (Torras-Claveria et al., 2012). Therefore, the concentration of this compound could be affected by many factors and the comprehension of its role during WS still remains a challenge.

In our study, the decreased SHA level in gr genotype, seem to indicate a lower activation of plant defenses, compared to rolc and wt, which could be related to the higher resistance of $g r$ plants toward WS; this suggestion, however, should be better investigated in order to assess the real potential of this genetic modification.

The role of SA and JA during WS conditions is currently under discussion. Our results suggested that the levels of these two phytohormone are not directly affected by water stress; however SA and JA could take part in the activation of early plant defenses, to be progressively depleted during stress exposure. Some studies indicate that high concentrations of SA could determine oxidative stress, thus leading to reduced abiotic stress tolerance, while, at moderate levels, SA is able to alleviate drought damages (Miura and Tada, 2014); in A. thaliana increased SA levels were related to an enhanced tolerance toward drought stress, as a consequence of the induction of $\mathrm{H}_{2} \mathrm{O}_{2}$ as signaling molecule (He et al., 2014). JA seems to be involved in ascorbate and glutathione metabolism, which is a key element in the cellular redox balance, and therefore could protect plants from water deficit-induced oxidative stress, as highlighted by malondialdehyde content and electrolyte leakage (Shan and Liang, 2010). Mahouachi et al., 2007 observed a transient accumulation of JA in papaya seedlings after 15 days of water deficit treatment, suggesting its role as a triggering signal for $A B A$ accumulation.

The application of three independent abiotic stresses and the analysis of key compounds involved in stress-response permitted 

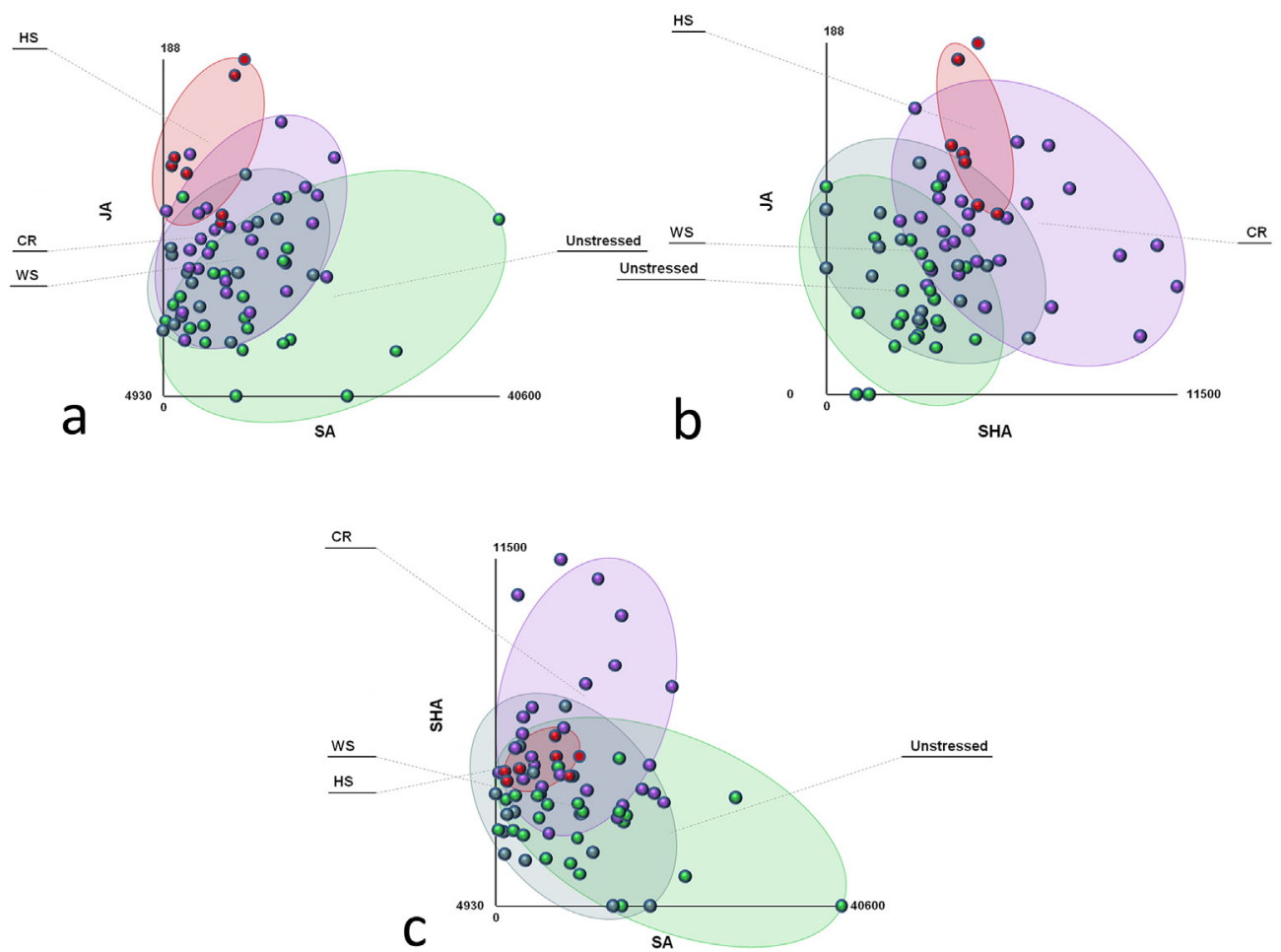

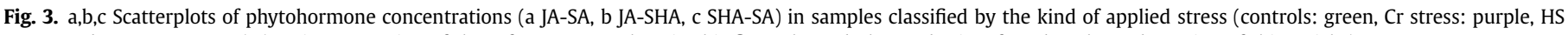
stress: red, WS stress: gray). (For interpretation of the references to colour in this figure legend, the reader is referred to the web version of this article.)

to highlight the main metabolic re-routing associated with each stress; as summarized in Fig. 3 a,b,c, Cr samples were characterized by medium-high concentrations of SHA, a clear indication of the enhanced production of secondary metabolites and particularly antioxidants; HS samples, instead, showed particularly high levels of JA (Fig 3 a,b), probably as a consequence of the peroxidation of lipids and the activation of the octadecenoic pathway induced by heat; WS samples displayed metabolite concentrations generally similar to the controls, indicating that the water deficit conditions involves different defense mechanisms and, probably, a more complex plant response.

\section{Conclusions}

The method presented in this work enabled the simultaneous and sensitive determination of three relevant plant metabolites, which proved to be useful in the assessment of the plant metabolic and biochemical status. The investigation of wild and genetically modified plants, exposed to three abiotic stresses, allowed the evaluation of the combined effect of transgenes and stresses on plant response mechanisms. The three genotypes showed to induce different responses during stress exposure; particularly, the insertion of the gr transgene showed to determine less changes in the phytohormonal pattern, suggesting the possibility of an advantage deriving from this genetic modification, especially in case of WS and $\mathrm{Cr}$ stresses, as already proposed in previous studies. These results could be useful for further investigations concerning the use of transgenic-stress resistant plants and in order to better understand the role of SA, JA and SHA in plant defense mechanisms.

\section{Contributions}

Elisa Scalabrin conducted the experiments, analyzed the data and wrote most of the manuscript. Marta Radaelli helped in plant sample preparation and revised the manuscript. Gabriele Capodaglio conceived and designed the experiments.

\section{Acknowledgments}

This work was supported by the PRIN grant number 20098TN4CY from the Italian Ministry of Education, University and Research (MIUR). The authors thank Marcello Buiatti and Patrizia Bogani for sample supply and Daniela Almansi for the English editing.

\section{Appendix A. Supplementary data}

Supplementary data related to this article can be found at http:// dx.doi.org/10.1016/j.plaphy.2016.02.040.

\section{References}

Ancillotti, C., Bogani, P., Biricolti, S., Calistri, E., Checchini, L., Ciofi, L., Gonnelli, C., Del Bubba, M., 2015. Changes in polyphenol and sugar concentrations in wild type and genetically modified Nicotiana langsdorffii Weinmann in response to water and heat stress. Plant Physiol. Biochem. 97, 52-61. http://dx.doi.org/10.1016/ j.plaphy.2015.09.012.

Bartwal, A., Mall, R., Lohani, P., Guru, S.K., Arora, S., 2013. Role of secondary metabolites and brassinosteroids in plant defense against environmental stresses. J. Plant Growth Regul. 32, 216-232. http://dx.doi.org/10.1007/s00344-0129272-X.

Bogani, P., Calistri, E., Biricolti, S., Ruffini, M., Turrini, A., Giovannetti, M., Buiatti, M., 2015. The rat glucocorticoid receptor integration in Nicotiana langsdorffii genome affects plant responses to abiotic stresses and to arbuscular mycorrhizal symbiosis. Plant Biotechnol. Rep. 9, 209-220. http://dx.doi.org/10.1007/ s11816-015-0358-3.

Bulgakov, V.P., Aminin, D.L., Shkryl, Y.N., Gorpenchenko, T.Y., Veremeichik, G.N., Dmitrenok, P.S., Zhuravlev, Y.N., 2008. Suppression of reactive oxygen species and enhanced stress tolerance in Rubia cordifolia cells expressing the rolc oncogene. Mol. Plant. Microbe. Interact. 21, 1561-1570. http://dx.doi.org/ 10.1094/MPMI-21-12-1561.

Clarke, S.M., Cristescu, S.M., Miersch, O., Harren, F.J.M., Wasternack, C., Mur, L.A., 2009. Jasmonates act with salicylic acid to confer basal thermotolerance in 
Arabidopsis thaliana. New Phytol. 182, 175-187. http://dx.doi.org/10.1111/j.14698137.2008.02735.x.

De Ollas, C., Hernando, B., Arbona, V., Gómez-Cadenas, A., 2013. Jasmonic acid transient accumulation is needed for abscisic acid increase in citrus roots under drought stress conditions. Physiol. Plant 147, 296-306. http://dx.doi.org/ 10.1111/j.1399-3054.2012.01659.x.

Del Bubba, M., Ancillotti, C., Checchini, L., Ciofi, L., Fibbi, D., Gonnelli, C., Mosti, S., 2013. Chromium accumulation and changes in plant growth, selected phenolics and sugars of wild type and genetically modified Nicotiana langsdorffii. J. Hazard. Mater 262, 394-403. http://dx.doi.org/10.1016/j.jhazmat.2013.08.073.

Fuoco, R., Bogani, P., Capodaglio, G., Del Bubba, M., Abollino, O., Giannarelli, S., Spiriti, M.M., Muscatello, B., Doumett, S., Turetta, C., Zangrando, R., Zelano, V., Buiatti, M., 2013. Response to metal stress of Nicotiana langsdorffii plants wildtype and transgenic for the rat glucocorticoid receptor gene. J. Plant Physiol. 170, 668-675. http://dx.doi.org/10.1016/j.jplph.2012.12.009.

Giannarelli, S., Muscatello, B., Bogani, P., Spiriti, M.M., Buiatti, M., Fuoco, R., 2010. Comparative determination of some phytohormones in wild-type and genetically modified plants by gas chromatography-mass spectrometry and highperformance liquid chromatography-tandem mass spectrometry. Anal. Biochem. 398, 60-68. http://dx.doi.org/10.1016/j.ab.2009.10.038.

He, Q., Zhao, S., Ma, Q., Zhang, Y., Huang, L., Li, G., Hao, L., 2014. Endogenous salicylic acid levels and signaling positively regulate Arabidopsis response to polyethylene glycol-simulated drought stress. J. Plant Growth Regul. 33, 871-880. http://dx.doi.org/10.1007/s00344-014-9438-9.

Intrieri, M.C., Buiatti, M., 2001. The horizontal transfer of Agrobacterium rhizogenes genes and the evolution of the genus Nicotiana. Mol. Phylogenet. Evol. 20 100-110. http://dx.doi.org/10.1006/mpev.2001.0927.

Kazan, K., 2015. Diverse roles of jasmonates and ethylene in abiotic stress tolerance. Trends Plant Sci. 20, 219-229. http://dx.doi.org/10.1016/j.tplants.2015.02.001.

Khalid, K.a., Teixeira, J.a., Cai, W., 2010. Water deficit and polyethylene glycol 6000 affects morphological and biochemical characters of Pelargonium odoratissimum ( L.). Sci. Hortic. Amst. 125, 159-166. http://dx.doi.org/10.1016/ j.scienta.2010.03.009.

Kiselev, K.V., Kusaykin, M.I., Dubrovina, A.S., Bezverbny, D.A., Zvyagintseva, T.N. Bulgakov, V.P., 2006. The rolC gene induces expression of a pathogenesisrelated beta-1,3-glucanase in transformed ginseng cells. Phytochemistry 67, 2225-2231. http://dx.doi.org/10.1016/j.phytochem.2006.07.019.

Kurepin, L.V., Dahal, K.P., Zhaman, M., Pharis, R.P., 2013. Interplay between Environmental Signals and Endogenous Salicylic Acid Concentration. In: Hayat, Shamsul, Ahmad, Aqil, Alyemeni, M.N. (Eds.), Salicylic Acid:Plant Growth and Development. Springer Science \& Business Media, pp. 61-82.

Lipiec, J., Doussan, C., Nosalewicz, A., Kondracka, K., 2013. Effect of drought and heat stresses on plant growth and yield: a review. Int. Agrophysics 27, 463-477. http://dx.doi.org/10.2478/intag-2013-0017.

Mahouachi, J., Arbona, V., Gómez-Cadenas, A., 2007. Hormonal changes in papaya seedlings subjected to progressive water stress and re-watering. Plant Growth Regul. 53, 43-51. http://dx.doi.org/10.1007/s10725-007-9202-2.

Maksymiec, W., 2007. Signaling responses in plants to heavy metal stress. Acta Physiol. Plant 29, 177-187. http://dx.doi.org/10.1007/s11738-007-0036-3.

Maksymiec, W., Wianowska, D., Dawidowicz, A.L., Radkiewicz, S., Mardarowicz, M., Krupa, Z., 2005. The level of jasmonic acid in Arabidopsis thaliana and Phaseolus coccineus plants under heavy metal stress. J. Plant Physiol. 162, 1338-1346. http://dx.doi.org/10.1016/j.jplph.2005.01.013.

Metwally, A., Finkemeier, I., Georgi, M., Dietz, K., 2003. Salicylic acid alleviates the cadmium toxicity in Barley seedlings. Plant Physiol. 132, 272-281. http:// dx.doi.org/10.1104/pp.102.018457.

Miura, K., Tada, Y., 2014. Regulation of water, salinity, and cold stress responses by salicylic acid. Front. Plant Sci. 5, 1-12. http://dx.doi.org/10.3389/ fpls.2014.00004.

Obata, T., Fernie, A.R., 2012. The use of metabolomics to dissect plant responses to abiotic stresses. Cell. Mol. life Sci. 69, 3225-3243. http://dx.doi.org/10.1007/ s00018-012-1091-5.

Pál, M., Horváth, E., Janda, T., Páldi, E., Szalai, G., 2005. Cadmium stimulates the accumulation of salicylic acid and its putative precursors in maize (Zea mays) plants. Physiol. Plant 125, 356-364. http://dx.doi.org/10.1111/j.1399- 3054.2005.00545.x

Pál, M., Szalai, G., Horváth, E., Janda, T., Páldi, E., 2002. Effect of salicylic acid during heavy metal stress. Acta Biol. szeg. 119-120.

Pan, Q., Zhan, J., Liu, H., Zhang, J., Chen, J., Wen, P., Huang, W., 2006. Salicylic acid synthesized by benzoic acid 2-hydroxylase participates in the development of thermotolerance in pea plants. Plant Sci. 171, 226-233. http://dx.doi.org/ 10.1016/j.plantsci.2006.03.012.

Ranaldo, M., Toscano, G., Radaelli, M., Scalabrin, E., Capodaglio, G., 2015. Nicotiana langsdorffii wild type and genetically modified exposed to chemical and physical stress: changes in element content. Int. J. Environ. Anal. Chem. 95, 349-365. http://dx.doi.org/10.1080/03067319.2015.1016013.

Rizhsky, L., Liang, H., Mittler, R., 2002. The combined effect of drought stress and heat shock on gene expression in tobacco. Plant Physiol. 130, 1143-1151. http:// dx.doi.org/10.1104/pp.006858.

Sánchez-Rodríguez, E., Moreno, D.a., Ferreres, F., Rubio-Wilhelmi, M.D.M., Ruiz, J.M. 2011. Differential responses of five cherry tomato varieties to water stress: changes on phenolic metabolites and related enzymes. Phytochemistry 72 723-729. http://dx.doi.org/10.1016/j.phytochem.2011.02.011.

Sano, H., Seo, S., Koizumi, N., Niki, T., Iwamura, H., Ohashi, Y., 1996. Regulation by cytokinins of endogenous levels of jasmonic and salicylic acids in mechanically wounded tobacco plants. Plant Cell Physiol. 37, 762-769. http://dx.doi.org/ 10.1093/oxfordjournals.pcp.a029011.

Scalabrin, E., Radaelli, M., Rizzato, G., Bogani, P., Buiatti, M., Gambaro, A Capodaglio, G., 2015. Metabolomic analysis of wild and transgenic Nicotiana langsdorffii plants exposed to abiotic stresses: unraveling metabolic responses. Anal. Bioanal. Chem. 407, 6357-6368. http://dx.doi.org/10.1007/s00216-015 8770-7.

Schmelz, E.A., Engelberth, J., Alborn, H.T., O' Donnell, P., Sammons, M., Toshima, H., Tumlinson III, J.H., 2003. Simultaneous analysis of phytohormones, phytotoxins, and volatile organic compounds in plants. Proc. Natl. Acad. Sci. U. S. A. 100 10552-10557. http://dx.doi.org/10.1073/pnas.1633615100.

Shan, C., Liang, Z., 2010. Jasmonic acid regulates ascorbate and glutathione metabolism in Agropyron cristatum leaves under water stress. Plant Sci. 178 130-139. http://dx.doi.org/10.1016/j.plantsci.2009.11.002.

Singh, V.P., Kumar, J., Singh, M., Singh, S., Prasad, S.M., Dwivedi, R., Singh, M.P.V.V.B., 2015. Role of salicylic acid-seed priming in the regulation of chromium (VI) and UV-B toxicity in maize seedlings. Plant Growth Regul. http://dx.doi.org/10.1007/ s10725-015-0076-4.

Torras-Claveria, L., Jáuregui, O., Codina, C., Tiburcio, A.F., Bastida, J., Viladomat, F., 2012. Analysis of phenolic compounds by high-performance liquid chromatography coupled to electrospray ionization tandem mass spectrometry in senescent and water-stressed tobacco. Plant Sci. 182, 71-78. http://dx.doi.org/ 10.1016/j.plantsci.2011.02.009.

Viehweger, K., 2014. How plants cope with heavy metals. Bot. Stud. 55, 35. http:// dx.doi.org/10.1186/1999-3110-55-35.

Wang, L.-J., Fan, L., Loescher, W., Duan, W., Liu, G.-J., Cheng, J.-S., Luo, H.-B., Li, S.-H., 2010. Salicylic acid alleviates decreases in photosynthesis under heat stress and accelerates recovery in grapevine leaves. BMC Plant Biol. 10, 34. http:// dx.doi.org/10.1186/1471-2229-10-34.

Warren, C.R., Aranda, I., Cano, F.J., 2012. Metabolomics demonstrates divergent responses of two Eucalyptus species to water stress. Metabolomics 8, 186-200. http://dx.doi.org/10.1007/s11306-011-0299-y.

Xiang, C., Oliver, D.J., 1998. Glutathione metabolic genes coordinately respond to heavy metals and jasmonic acid in Arabidopsis. Plant Cell 10,1539-1550. http:// dx.doi.org/10.1105/tpc.10.9.1539.

Yang, Y., Qi, M., Mei, C., 2004. Endogenous salicylic acid protects rice plants from oxidative damage caused by aging as well as biotic and abiotic stress. Plant J. 40 909-919. http://dx.doi.org/10.1111/j.1365-313X.2004.02267.x.

Yordanov, I., Velikova, V., Tsonev, T., 2000. Plant responses to drought, acclimation and stress tolerance. Photosynthetica 38, 171-186. http://dx.doi.org/10.1023/A: 1007201411474

Zawoznik, M.S., Groppa, M.D., Tomaro, M.L., Benavides, M.P., 2007. Endogenous salicylic acid potentiates cadmium-induced oxidative stress in Arabidopsis thaliana. Plant Sci. 173, 190-197. http://dx.doi.org/10.1016/ j.plantsci.2007.05.004. 